Cite this: RSC Adv., 2014, 4, 14915

\title{
Ion responsive near-IR BODIPY dyes: two isomers, two different signals $\uparrow$
}

Received 4th February 2014

Accepted 6th March 2014

\author{
Tugba Ozdemir, ${ }^{a}$ Ziya Kostereli, ${ }^{\text {b }}$ Ruslan Guliyev, ${ }^{\text {b }}$ Soydan Yalcin, ${ }^{c}$ Yavuz Dede \\ and Engin U. Akkaya*ab
}

DOI: $10.1039 / c 4 r a 00989 d$

www.rsc.org/advances

Tetrastyryl-substituted BODIPY dyes are likely to evolve into a new class of near IR fluorophores. In this work we demonstrate that 1,7 and 3,5-positions show marked differences in charge transfer characteristics. Using a $\mathrm{Hg}(\mathrm{II})$ selective ligand, the signal transduction potentials were explored: one isomer shows a large blue shift in electronic absorption spectrum, while the other just shows an intensity increase in the emission spectrum. Electronic structure calculations were undertaken to elucidate the reasons for different signals on metal ion binding in relation to core BODIPY properties.

Fluorescent dyes exhibiting near-IR absorption and emission are rare, ${ }^{\mathbf{1}}$ and considering their potential utility in many fields such as biological imaging, ${ }^{2}$ photodynamic therapy, ${ }^{3}$ light harvesters, ${ }^{\mathbf{4}}$ solar cells, ${ }^{5}$ there is a strong motivation for developing such organic compounds. Longer wavelength emitting fluorescent dyes are relatively free from background signals, resulting either from Rayleigh scattering or autofluorescence in biological media. ${ }^{6}$ BODIPY dyes are truly unique in their capacity for chemical modifications at all positions of the parent dye. ${ }^{7}$ Derivatization at the $1,3,5,7$ positions via Knoevenagel condensation results in large bathochromic shifts, pushing the $\mathrm{S}_{0} \rightarrow \mathrm{S}_{1}$ absorption band towards near IR. Recently, tetrastyryl BODIPY derivatives were successfully synthesized and shown to be stable chromophores with reasonable fluorescence quantum yields. Following our initial report, ${ }^{8}$ quadruple Knoevenagel condensation on 1,3,5,7-tetramethyl BODIPY dyes has become a well-established protocol and even controlled sequential reaction of methyl groups at these positions with different aldehydes was demonstrated. ${ }^{9}$ Tetrastyryl-BODIPY's

${ }^{a}$ UNAM-National Nanotechnology Research Center, Bilkent University, 06800 Ankara, Turkey.E-mail: eua@fen.bilkent.edu.tr

${ }^{b}$ Department of Chemistry, Bilkent University, 06800 Ankara, Turkey

${ }^{c}$ Department of Chemistry, Gazi University, Ankara, 06500, Turkey

$\dagger$ Electronic supplementary information (ESI) available: Experimental procedures, structural proofs, additional spectroscopic data, ITC details and computational studies are provided. See DOI: 10.1039/c4ra00989d are now very well-positioned to become a structurally flexible class of near IR dyes.

In the present work, our aim was to explore the ion signaling potential of the dyes with charge donor ligand placed in conjugation at 1,7 versus 3,5 positions of the BODIPYs, i.e., four isomeric tetrastryryl BODIPY compounds which were functionalized with $\mathrm{Hg}(\mathrm{II})$ selective dithiaazacrown ligands (Scheme 1). These derivatives were readily synthesized based on already established protocols (ESI, Scheme S1 $\dagger$ ). Appropriate aromatic aldehydes undergo Knoevenagel reaction in the presence of acetic acid and piperidine using a Dean-Stark trap. Besides, being a convenient and efficient reaction, Knoevenagel condensation gives reasonable yields.

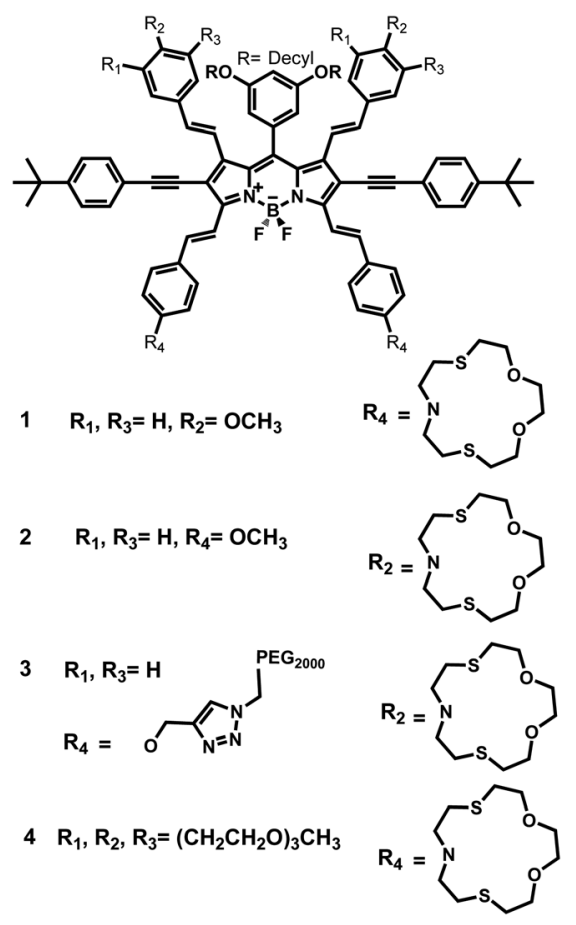

Scheme 1 Structures of target compounds 1-4. 

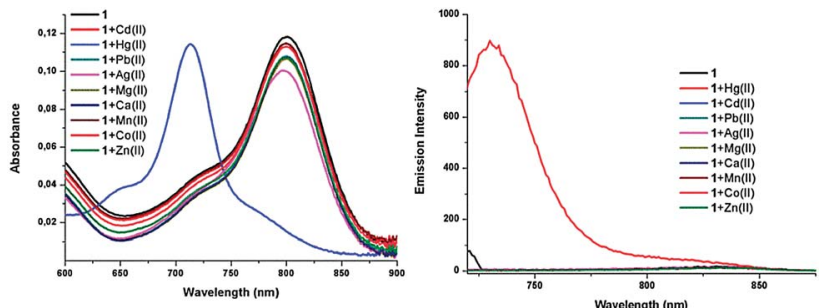

Fig. 1 Electronic absorption and emission spectra of $1(0.5 \mu \mathrm{M})$ in THF in absence and presence of various metal ions. Added metal ion concentrations were $5 \mu \mathrm{M}$. Excitation wavelength was $710 \mathrm{~nm}$.

Investigation of the electronic absorption and fluorescence spectra of the isomeric probes reveals a very different spectral response pattern regarding ion response. In electronic absorption spectra an impressive hypsochromic shift was observed for 1 in response to $\mathrm{Hg}$ (II) ions (Fig. 1). Initially, the absorbance band has a peak centered at $800 \mathrm{~nm}$ in THF and upon the addition of $\mathrm{Hg}$ (II) ions, a blue shift is observed and the peak maximum moves to $715 \mathrm{~nm}$. Also, when excited at $710 \mathrm{~nm}$, the emission intensity is essentially switched on, from a non-fluorescent state to a brightly fluorescent metal ion complex. Since the ligand of our choice, the dithiaazacrown, is known to be highly $\mathrm{Hg}$ (II) selective, ${ }^{\mathbf{1 0}}$ other metal ions do not interfere and thus, there is no change in either electronic absorption or fluorescence spectra under the experimental conditions of the study. Thus, we have a highly selective chemosensor for $\mathrm{Hg}$ (II) operating at near IR region of the spectrum.

Surprisingly, electronic absorption spectrum of isomer 2 shows a peak at $745 \mathrm{~nm}$ and the addition of $\mathrm{Hg}$ (II) ions does not alter the electronic absorption characteristics. However, just like the isomer 1, emission intensity of 2 shows remarkable increase (excitation wavelength is $725 \mathrm{~nm}$ ). Similarly, as a wellknown $\mathrm{Hg}$ (II) specific ligand, dithiaazacrown moiety does not respond to other metal ions (Fig. 2). Data for competition/ interference experiments with isomers 1 and 2 (using $\mathrm{Hg}$ (II) and selected metal ions) are given in ESI. $\dagger$ Water soluble fluorescent probes are especially in high demand for biological applications. To that end, in order to enhance water solubility, polyethylene glycol (PEG) and triethylene glycol groups were attached to BODIPY unit via Knoevenagel condensation reactions with adequate yields, and compound $\mathbf{3}$ and 4 were obtained. Electronic absorption and emission spectra in
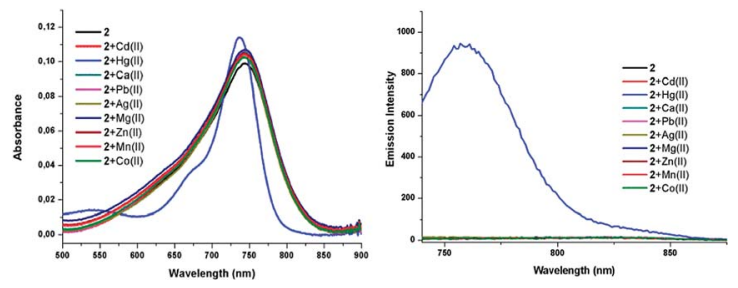

Fig. 2 Electronic absorption and emission spectra of $2(1.0 \mu \mathrm{M})$ in THF in absence and presence of various metal ions. Added metal ion concentrations were $10 \mu \mathrm{M}$. Excitation wavelength is $725 \mathrm{~nm}$.
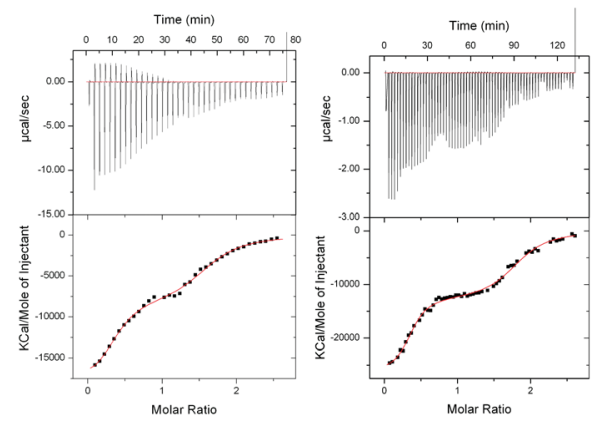

Fig. 3 Calorimetric binding isotherm for the compound 3 (right, 0.4 $\mathrm{mM} 3$ titrated with $\left.5 \mathrm{mM} \mathrm{Hg}\left(\mathrm{ClO}_{4}\right)_{2}\right)$ and 4 (left, $0.14 \mathrm{mM} 4$ titrated with $\left.2 \mathrm{mM} \mathrm{Hg}\left(\mathrm{ClO}_{4}\right)_{2}\right)$ in acetonitrile.

aqueous solutions $10 \mathrm{mM}$ HEPES- $\mathrm{CH}_{3} \mathrm{CN}(50: 50, \mathrm{v} / \mathrm{v}, \mathrm{pH}=7.2$, $25{ }^{\circ} \mathrm{C}$ ) of probes showed results in accordance with the organic soluble counterparts (see $\mathrm{ESI} \dagger$ ).

To determine binding constants, isothermal titration calorimetry (ITC) was performed. The inflection point in the titration curve (heat $v s$. molar ratio of metal ion to ligand), the stoichiometry of complexation can be determined. Table S2 in the $\mathrm{ESI} \dagger$ describes the binding characteristics for isomer 3 and 4. The results showed these water soluble isomeric compounds showed 1:2 complex formation in acetonitrile as expected (Fig. 3).

Significantly different spectral responses of $\mathbf{1}$ and 2 to $\mathrm{Hg}$ (II) binding indicates an electronic structure difference among the two probes. Theoretical studies employing the Time-Dependent (TD) formalism of Density Functional Theory (DFT) were performed to gain insight into the excitation characteristics and fluorescence turn on of $\mathbf{1}$ and 2. The presumed impact of structural differences of $\mathbf{1}$ and $\mathbf{2}$ on emission characteristics were studied by constructing the models 1a and 2a (Scheme 2). Effect of metal binding was investigated via protonation at $\mathrm{N}_{1}$ (1aH and 2aH). TD-DFT results (Fig. $\mathrm{S} 29$ and $\mathrm{S} 30$ and Table $\mathrm{S} 3 \dagger$ ) are in good agreement with the spectroscopic measurements and reveal that the fluorescence turn on is due to extinguishing the electron density on $\mathrm{N}_{1}$ lone pair upon metal binding. Note that the dative $\mathrm{N}_{1}$ : $\rightarrow \mathrm{Hg}(\mathrm{II})$ bonding diminishes the $\mathrm{n} \rightarrow \pi^{*}$ contribution to the $S_{0} \rightarrow S_{1}$ excitation. Consequently any input (metal or proton) able to utilize the $\mathrm{N}_{1}$ lone pair in binding is well suited for fluorescence turn on. Understanding different spectral responses requires a simpler, yet fundamental
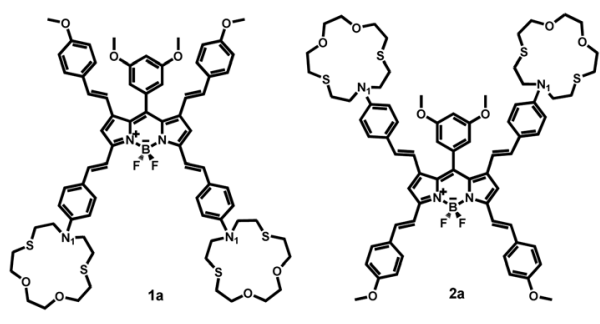

Scheme 2 Computational models. 
Table 1 MO plots and energies (eV) of BODIPY and styryl-substituted derivatives at UB3LYP/cc-pVTZ//6-31G(d) level of theory

MO

approach. Considering the decisive role of position of substitution on BODIPY core, we compared the frontier MO energies of di- and tetra-styryl substituted BODIPY derivatives (Table 1). Remarkably, 3,5 substitution was superior to 1,7 in altering the HOMO - LUMO gap of BODIPY, mainly by destabilization of HOMO. Improvement by obtaining the tetra-styryl derivative was minor when compared to 3,5-distyryl BODIPY. Thus, 3 and 5 positions are more important when perturbations to the electronic structure are sought however, this observation can be traced back to fundamental features of BODIPY core.

Comparison of MO plots for di- and tetra-styryl derivatives (Table 1) reveals that extension of the $\pi$-system at 3,5-positions preserves the character of HOMO of BODIPY core whereas 1,7modification yields a HOMO with a different character than BODIPY HOMO. Moreover, BODIPY HOMO hosts more electronic density at 3 and 5 positions than 1 and 7 which gives rise to an increased Coulombic repulsion that destabilizes the HOMO. The aforementioned difference in destabilization of HOMO (Table 1) is thus justified. Consequently, electronic structure of 1a upon proton or cation binding experiences larger perturbations than $\mathbf{2 a}$ and this is also the case for $\mathbf{1}$ when compared to 2 . Therefore the discrepancy in the absorption shifts of 1 and 2 is mainly due to the $\pi$-system of BODIPY core treating the charge injections unequally, i.e. as dictated by the spatial distribution of HOMO.

In conclusion, we demonstrated that tetrastyryl-BODIPY dyes can be derivatized to yield ion responsive compounds functioning in the near IR wavelengths. Also, we provided the first examples of divergent ion response resulting from a difference in the locations of styryl-linked donor groups on the BODIPY core and the impact of their changing charge donation properties on ion binding. Principles underlying different spectroscopic properties and fluorescence turn on response are explained via orbital analysis. It is very likely that these stable and near IR emissive probes, or others built upon the ideas developed here will be quickly added to growing arsenal of ion probes successfully interrogating cellular events, or monitoring environmental parameters.

\section{Acknowledgements}

The authors gratefully acknowledge support from TUBITAK in the form of grant. Y.D. thanks TUBITAK (110T647) and computing resources of TR-GRID. S.Y. thanks TUBITAK for scholarship.

\section{Notes and references}

1 (a) J. Fabian, H. Nakazumi and M. Matsuoka, Chem. Rev., 1992, 92, 1197; (b) K. Kiyose, H. Kojima and T. Nagano, Chem. -Asian J., 2008, 50, 506; (c) A. Loudet, R. Bandichhor, K. Burgess, A. Palma, S. O. McDonnell, M. J. Hall and D. F. O'Shea, Org. Lett., 2008, 10, 4771; (d) S. Goeb and R. Ziessel, Org. Lett., 2007, 9, 737; (e) K. Umezawa, Y. Nakamura, H. Makino, D. Citterio and K. J. Suzuki, J. Am. Chem. Soc., 2009, 130, 1550; (f) Y. Xiao, F. Liu and X. Qian, Chem. Commun., 2005, 239.

2 (a) V. Ntziachristos, C. Bremer and R. Weissleder, Eur. J. Radiol., 2003, 13, 195; (b) J. V. Frangioni, Curr. Opin. Chem. Biol., 2003, 7, 626; (c) P. Li, X. Duan, Z. Chen, Y. Liu, T. Xie, L. Fang, X. Li, M. Yinb and B. Tang, Chem. Commun., 2011, 47, 7755; (d) Y. Koide, Y. K. Hanaoka, W. Piao, M. Kusakabe, N. Saito, T. Terai, T. Okabe and T. Nagano, J. Am. Chem. Soc., 2012, 134, 5029; (e) N. Jiang, J. Fan, T. Liu, J. Cao, B. Qiao, J. Wang, P. Gao and X. Peng, Chem. Commun., 2013, 49, 10620; (f) Z. Guo, S. Park, J. Yoon and I. Shin, Chem. Soc. Rev., 2014, 43, 16-29.

3 (a) S. W. Young, K. W. Woodburn, M. Wrigth, T. D. Mody, Q. Fan, J. L. Sessler, W. C. Dow and R. A. Miller, Photochem. Photobiol., 1996, 63, 892; (b) X. Tan, S. Luo, D. Wang, Y. Su, T. Cheng and C. Shi, Biomaterials, 2012, 33, 2230; (c) Y. Yang, Q. Guo, H. Chen, Z. Zhou, Z. Guo and Z. Shen, Chem. Commun., 2013, 49, 3940; (d) S. Erbas, A. Gorgulu, M. Kocakusakogullari and E. U. Akkaya, Chem. Commun., 2009, 4956.

4 (a) Z. Kostereli, T. Ozdemir, O. Buyukcakir and E. U. Akkaya, Org. Lett., 2012, 14, 3636; (b) F. Sozmen, B. S. Oksal, O. A. Bozdemir, O. Buyukcakir and E. U. Akkaya, Org. Lett., 
2012, 14, 5286; (c) A. Harriman, L. J. Mallon, S. Goeb and R. Ziessel, Phys. Chem. Chem. Phys., 2007, 9, 5199; (d) R. Guliyev, A. Coskun and E. U. Akkaya, J. Am. Chem. Soc., 2009, 131, 9007-9013.

5 (a) S. Kolemen, Y. Cakmak, S. Erten-Ela, Y. Altay, J. Brendel, M. Thelakkat and E. U. Akkaya, Org. Lett., 2010, 12, 3812; (b) J. He, G. Banko, F. Korodi, T. Polivka, R. Lomoth, B. Akermark, L. Sun, A. Hagfeldt and V. Sundstrom, J. Am. Chem. Soc., 2002, 124, 4922.

6 R. Weissleder, Nat. Biotechnol., 2001, 19, 316.
7 (a) A. Loudet and K. Burgess, Chem. Rev., 2007, 107, 4891; (b) G. Ulrich, R. Ziessel and A. Harriman, Angew. Chem., Int. Ed., 2008, 47, 1184.

8 O. Buyukcakir, O. A. Bozdemir, S. Kolemen, S. Erbas and E. U. Akkaya, Org. Lett., 2009, 11, 4644.

9 (a) S. Zhu, J. Zhang, G. Vegesna, A. Tiwari, F.-T. Luo, M. Zeller, R. Luck, H. Li, S. Greena and H. Liu, RSC Adv., 2012, 2, 404; (b) T. Bura, P. Retailleau, G. Ulrich and R. Ziessel, J. Org. Chem., 2011, 76, 1109.

10 S. Atilgan, I. Kutuk and T. Ozdemir, Tetrahedron Lett., 2010, 51, 892 . 